\title{
Micro-cavities based on width modulated SOI waveguides
}

Meister, Stefan, Al-Saadi, Aws, Franke, Bülent, Mahdi, Shaimaa, Szczambura, Miroslaw, et al.

Stefan Meister, Aws Al-Saadi, Bülent A. Franke, Shaimaa Mahdi, Miroslaw Szczambura, Berndt Kuhlow, Ulrike Woggon, Lars Zimmermann, Harald H. Richter, David Stolarek, Sigurd K. Schrader, Hans J. Eichler, "Micro-cavities based on width modulated SOI waveguides," Proc. SPIE 7943, Silicon Photonics VI, 79430T (17 January 2011); doi: 10.1117/12.875652

SPIE. Event: SPIE OPTO, 2011, San Francisco, California, United States 


\title{
Micro-cavities based on width modulated SOI waveguides
}

\author{
Stefan Meister*a , Aws Al-Saadi ${ }^{\mathrm{a}}$, Bülent A. Franke ${ }^{\mathrm{a}}$, Shaimaa Mahdi ${ }^{\mathrm{a}}$, Miroslaw Szczambura ${ }^{\mathrm{a}}$, \\ Berndt Kuhlow ${ }^{\mathrm{a}}$, Ulrike Woggon ${ }^{\mathrm{a}}$, Lars Zimmermann ${ }^{\mathrm{b}}$, Harald H. Richter ${ }^{\mathrm{b}}$, David Stolarek ${ }^{\mathrm{b}}$, \\ Sigurd K. Schrader ${ }^{\mathrm{c}}$, Hans J. Eichler ${ }^{\mathrm{a}}$ \\ ${ }^{a}$ Technische Univ. Berlin, Institut für Optik und Atomare Physik, ER1-1, \\ Str. des 17. Juni 135, 10623 Berlin, Germany; \\ ${ }^{\mathrm{b}}$ Innovations for High Performance microelectronics (IHP) GmbH, Im Technologiepark 25, \\ 15236 Frankfurt (Oder), Germany; \\ ${ }^{\mathrm{c}}$ Technische Fachhochschule Wildau, Institut für Plasma- und Lasertechnik, Bahnhofstr. \\ 15745 Wildau, Germany
}

\begin{abstract}
We have designed, fabricated and investigated one-dimensional (1D) micro-cavities in Silicon-on-Insulator (SOI) waveguides. The single mode waveguides are fabricated in a $220 \mathrm{~nm}$ silicon device layer. The 1D micro-cavities in Fabry-Perot structure consist of two Bragg-mirror regions formed by a sinusoidal modulation of the waveguide width. The mirror regions are separated by a sub-micron spacer.

The SOI photonic structures are produced in a CMOS environment using $248 \mathrm{~nm}$ DUV lithography. The waveguides as well as the width modulated mirror regions are designed using a single mask and are fabricated in a shallow trench process.

The transmission spectra of these width modulated micro-cavities with different mirror reflectivities and cavity lengths are investigated. Q-factors up to 855 could be observed at $1550 \mathrm{~nm}$ wavelength with low insertion loss of $1.9 \mathrm{~dB}$.

The width modulated micro-cavities, including the mirror regions, have lengths of less than 20 microns and widths of maximum $450 \mathrm{~nm}$. These small foot-print cavities act as band pass filters and can be used as resonators for laser or electro-optic modulation of light.
\end{abstract}

Keywords: silicon photonics, SOI waveguides, micro-cavity, micro-resonators, band pass filter, CMOS

\section{INTRODUCTION}

Silicon photonics, especially based on silicon-on-insulator (SOI) attracted great attention in the last years. With an intrinsic bandgap of $1.1 \mathrm{eV}$ silicon is transparent at wavelengths typically used for optical communication transmission, i.e. $1270 \mathrm{~nm}-1625 \mathrm{~nm}$. In addition, the possibility to produce silicon photonics devices in a complementary metal oxide semiconductor (CMOS) environment offers an opportunity for very low-cost photonic solutions. Furthermore, the ability to integrate silicon photonic devices with electronic control functions opens a wide range of applications from telecommunications to chip-to-chip interconnects ${ }^{1}$, on-chip communication as well as possible applications in emerging areas such as optical sensing and biomedical devices².

Ultrasmall cavities play an important role in photonic integrates circuits (PIC) by controlling light for trapping and emission of photons ${ }^{3}$, lasing ${ }^{4}$, switching, and optical filtering. Most popular structures are ring resonators ${ }^{5}$ and photonic crystal micro-cavities ${ }^{6,7}$. One-dimensional (1D) micro-cavities have a very small footprint and are, therefore, well suited for highly dense packaging.

In this paper, the characteristics of 1D micro-cavities based on width modulated SOI waveguides are investigated, which are fabricated in a CMOS environment. The micro-cavities act as band pass filters. The filter design parameters, e.g. number of modulations, cavity length, etc., are systematically changed to investigate the limits of such 1D microcavities.

*smeister@physik.tu-berlin.de; phone: +49-30-31426227; fax: +49-30-31426888

Silicon Photonics VI, edited by Joel A. Kubby, Graham T. Reed, Proc. of SPIE Vol. 7943,

79430T · (c) 2011 SPIE · CCC code: 0277-786X/11/\$18 · doi: 10.1117/12.875652

Proc. of SPIE Vol. 7943 79430T-1 


\title{
2. DESIGN OF THE MICRO-CAVITIES
}

The filters here are designed for the telecommunication wavelengths range around $1550 \mathrm{~nm}$. The 1D micro-cavities in Fabry-Perot structure consist of two Bragg-mirror regions separated by a sub-micron spacer. The two mirror regions are formed by modulation of the waveguide width. In the design, the mirror regions are defined by pairs of so called halfholes on both sides of the waveguide. The centers of the holes are placed on the edges of the waveguides. Figure 1 shows the basic structure and parameters of the width modulated micro-cavities.

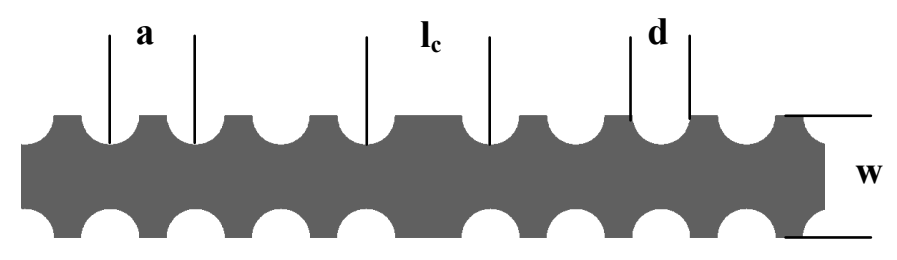

\author{
Constant parameters \\ Max. waveguide width $\mathrm{w} \sim 450 \mathrm{~nm}$ \\ Waveguide height $\mathrm{h} \sim 220 \mathrm{~nm}$ \\ Lattice constant a $\sim 390 \mathrm{~nm}$
}

Figure 1. Basic design structure and parameters of the width modulated micro-cavities.

The lattice constant a and cavity length $l_{\mathrm{c}}$ are both measured from center to center between neighboring half-holes. The rectangular waveguides (also called nanowires) are fabricated in the $220 \mathrm{~nm}$ thick silicon device layer of the SOI substrate. Therefore, the waveguide height is fixed to $\mathrm{h}=220 \mathrm{~nm}$. The micro-cavities are written in singlemode waveguides of $450 \mathrm{~nm}$ width.

The fixed lattice constants $\mathrm{a}=390 \mathrm{~nm}$ have been chosen for all mirrors, see figure 1 . Different half-hole diameters between $\mathrm{d}=160 \mathrm{~nm}$ and $\mathrm{d}=220 \mathrm{~nm}$ were realized in the mask design. The micro-cavities were designed with two mirror regions of 10 or 20 half-holes pairs, e.g. $20+20$ half-holes pairs. The half-hole diameter as well as the number of half-holes will change the mirror reflectivity and therefore the filter bandwidth. For the first order cavity filters, three different cavity lengths $1_{c}$ were introduced, ranging from $l_{c}=570 \mathrm{~nm}$ to $600 \mathrm{~nm}$ in steps of $15 \mathrm{~nm}$. The cavity length has an influence on the wavelength of the transmission peak.

\section{FABRICATION OF WIDTH MODULATED MICRO-CAVIIIES}

The SOI photonic structures are produced in a $0.13 \mu \mathrm{m}$ SiGe BiCMOS environment at IHP GmbH, Frankfurt (Oder), Germany. The photonic devices were fabricated in $200 \mathrm{~mm}$ SOI wafers using a $248 \mathrm{~nm}$ DUV lithography. Waveguides as well as the width modulated micro-cavities are designed using a single mask and fabricated in a shallow trench process. The waveguides as well as the width modulated micro-cavities are etched completely down to the BOX layer, which has a thickness of $2 \mu \mathrm{m}$.

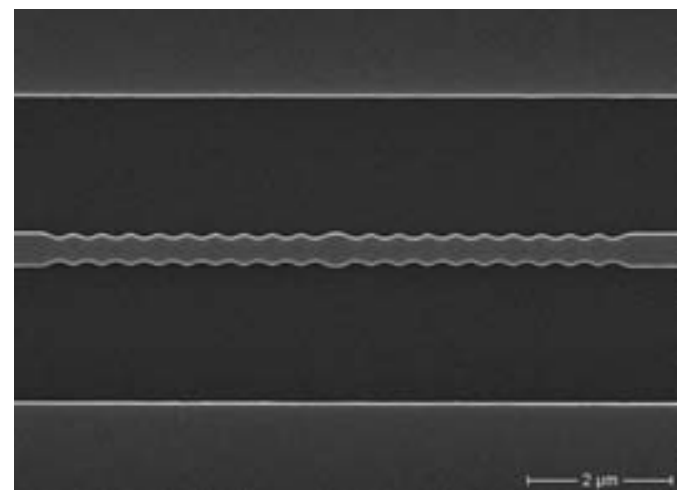

Figure 2. SEM image of a 1D width modulated micro-cavity with 10 designed half-hole pairs in each of the two mirror regions $(10+10)$ and a sub-micron spacer in the center of the cavity. 
Physically, the waveguides were formed by inserting two parallel running grooves into the Silicon surface, which distance to each other define the waveguide width. These lateral trenches are wide enough to prevent waveguide leakage into the surrounding intact top SOI areas.

The SEM image in figure 2 shows a top view of a 1D micro-cavity. The two width modulated mirror regions were designed with 10 half-holes each. Between the mirror regions is seen the slightly wider spacer. Also seen are the two etched trenches forming the waveguide. The higher magnification in figure 3 shows that the waveguide between the designed half-holes are rounded due to fabrication process leading to a sinusoidal modulation of the waveguide width. Also the sub-micron spacer in the center of the images is rounded. The designed half-hole diameters of the structure shown in figure $3 \mathrm{a}$ was $160 \mathrm{~nm}$, which results in a slightly width modulated micro-cavity. The $220 \mathrm{~nm}$ designed halfhole diameters of the structure shown in figure $3 \mathrm{~b}$ leads to a comparable strong modulation of the waveguide. In both cases, the modulation of the waveguide width is sinusoidal.
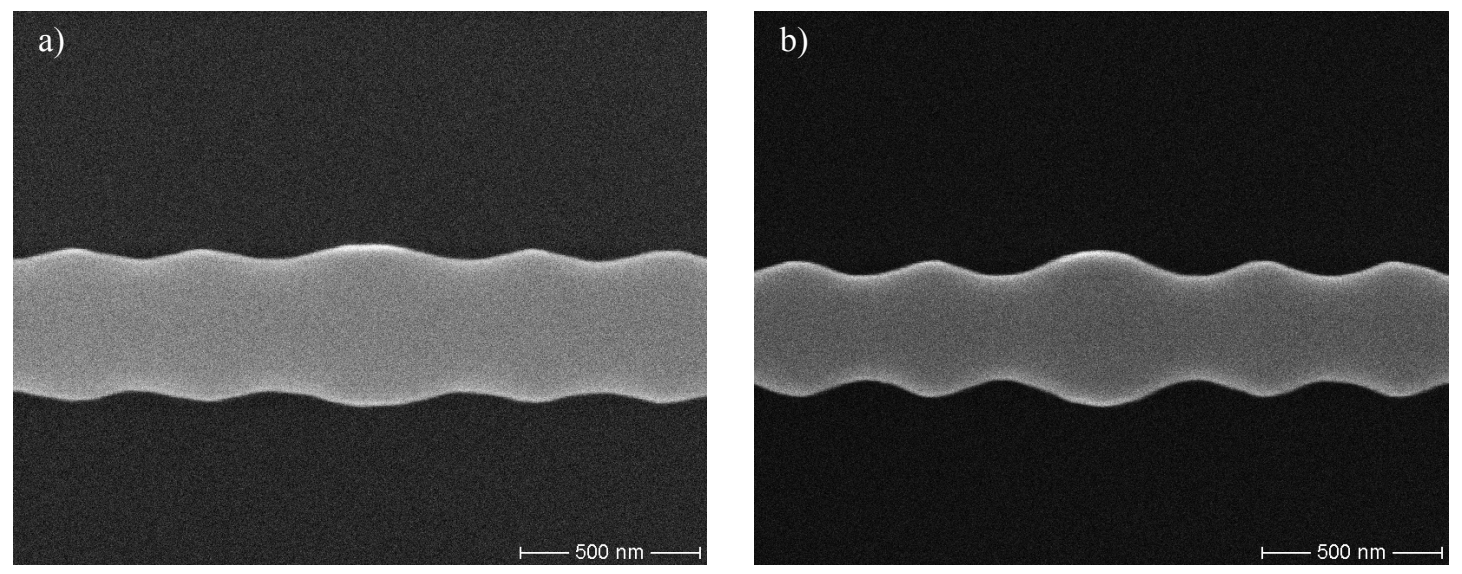

Figure 3. SEM images of the center part of width modulated micro-cavities, including the sub-micron spacer, etched in a SOI waveguides. a) The designed half-hole diameter was $160 \mathrm{~nm}$. b) The designed half-hole diameter was $220 \mathrm{~nm}$.

\section{EXPERIMENTAL DETAILS FOR OPTICAL CHARACTERISATION}

Figure 4a shows the experimental setup for optical characterization of the 1D width modulated micro-cavities. The samples to be tested were fixed on a specially constructed vacuum chuck with integrated temperature control on top of a translation stage. Coupled SLEDs, providing a broad spectral range of $1200 \mathrm{~nm}-1700 \mathrm{~nm}$, serve as white light (WL) sources for transmission measurements. The light was coupled into the SOI waveguides via a tapered optical fiber $(\mathrm{OZ}$ Optics), see figure $4 \mathrm{~b}$. The taped fiber has a focal diameter of $2.5 \mu \mathrm{m}$ and a working distance of $14 \mu \mathrm{m}$.

a)

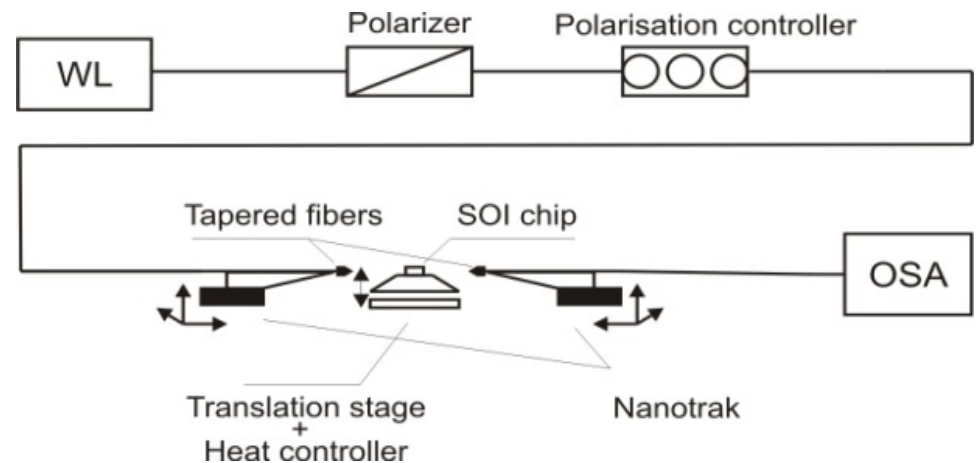

b)

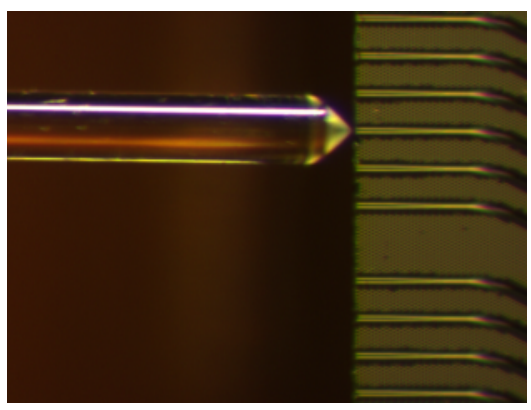

Figure 4. a) Experimental setup for optical characterization of the width modulated micro-cavities. b) Tapered fiber which couples the light in a SOI waveguide. 
TE polarization (E-field along the wider waveguide side) is necessary to excite the cavity modes. In order to achieve TE polarization at the waveguide input, a fiber optical polarizer followed by a polarization controller ${ }^{8}$ was inserted. The output light of the SOI waveguide was collected by a second (identical) tapered fiber. Both tapered fibers were mounted on a piezo controlled translation stage (NanoMax TS3), respectively. The output light was measured by an Optical Spectrum Analyzer (OSA). A resolution bandwidth of $0.2 \mathrm{~nm}$ was selected in the measurements.

\section{RESULTS AND DISCUSSION}

As reference for the transmission measurements, dummy waveguides without micro-cavities were used, which had the same physical dimensions as the waveguides carrying the micro-cavities. Most of the induced losses can be assigned to coupling losses from mode mismatch between the fibers and the SOI waveguide.

The transmission of width modulated micro-cavities, formed by two identical Bragg-mirror regions are investigated in dependence on the number of the designed half-holes. Furthermore, the cavities are of first order, which means the cavity length $l_{\mathrm{c}}$ has the shortest possible length for a certain transmission peak wavelength. In a classical Fabry-Perot, this would correspond to an optical spacer thickness of $\lambda / 2$, if $\lambda$ is the center wavelength at the transmission band of the filter. Figure 5 shows the transmission spectrum of a width modulated micro-cavity filter with 10+10 designed half-holes, in a) logarithmic and b) linear scale, respectively. The diameter of the designed half-holes was $\mathrm{d}=180 \mathrm{~nm}$ and the cavity length $l_{c}=600 \mathrm{~nm}$.

a)

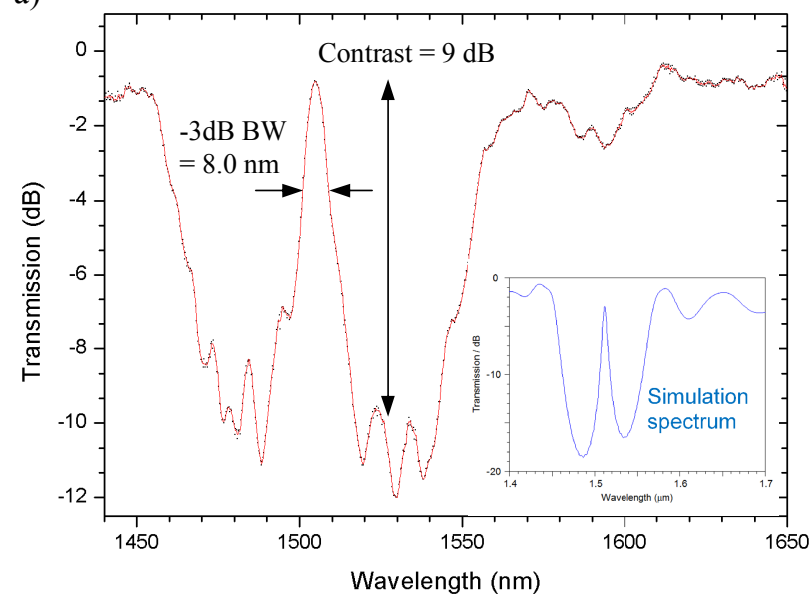

b)

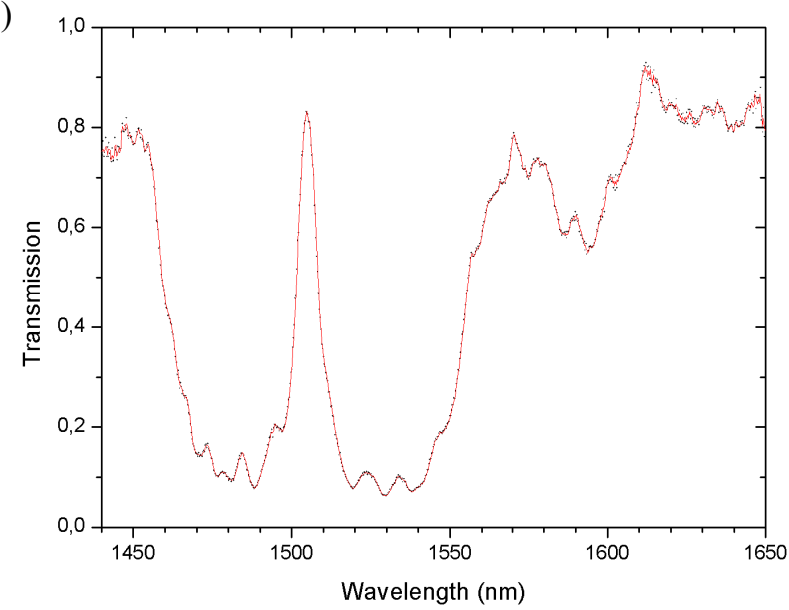

Figure 5. Transmission band of a width modulated micro-cavity filter with 10+10 designed half-holes a) Logarithmic scale, b) linear scale. The hole diameter was $200 \mathrm{~nm}$ and the cavity length was $1_{\mathrm{c}}=570 \mathrm{~nm}$.

The transmission spectrum of the micro-cavity exhibits a stop band from $1460 \mathrm{~nm}$ to $1550 \mathrm{~nm}$ with low transmission. Due to the relative low index contrast in the mirror regions, the width of the low transmission range is relative narrow. The transmission peak of the filter is located in the center of the stop band. The $-3 \mathrm{~dB}$ bandwidth of the transmission peak is $8.0 \mathrm{~nm}$, which corresponds to a quality factor of $\mathrm{Q}=188$. The filter contrast is about $9 \mathrm{~dB}$ for wavelengths $10 \mathrm{~nm}$ off to the filter peak. The $0.8 \mathrm{~dB}$ insertion loss of the filter is very low.

The insertion in figure 5a shows a simulated spectrum of this filter structure. In the simulation, using a Finite-DifferenceTime-Domain (FDTD) method, the chosen grid introduces virtual roughness to the sinusoidal width modulated waveguides, which lead to calculated scattering losses. Hence, the simulated filter losses are higher than the measured losses in the real waveguides.

If the number of designed half-holes in the mirrors is increased to 20 , the reflectivity of the Bragg mirrors will be higher. The transmission spectrum of a $20+20$ width modulated micro-cavity is shown in figure 6 , in a) logarithmic and b) linear scale, respectively. The diameter of the designed half-holes was $d=180 \mathrm{~nm}$ and the cavity length $1_{c}=600 \mathrm{~nm}$. 
Due to the higher mirror reflectivity the $-3 \mathrm{~dB}$ bandwidth of this filter is narrowed to $1.8 \mathrm{~nm}$, which corresponds to a quality factor of $\mathrm{Q}=855$. The filter contrast is increased to $13 \mathrm{~dB}$ for wavelengths $6 \mathrm{~nm}$ off the filter peak. The $1.9 \mathrm{~dB}$ insertion loss is low for such a filter.

a)

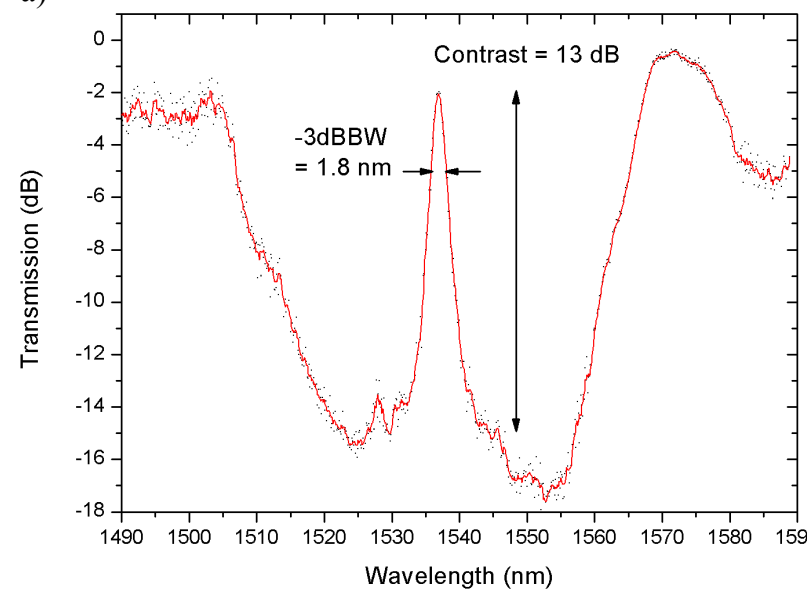

b)

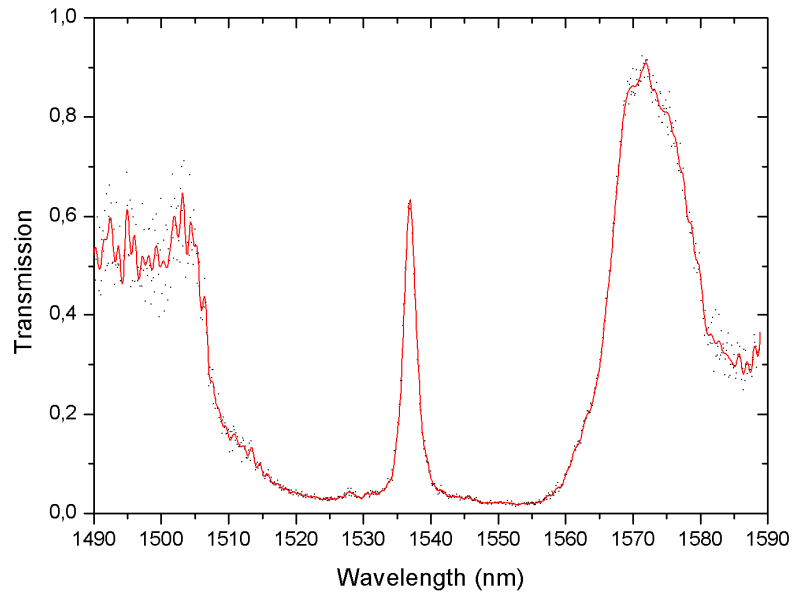

Figure 6. Transmission band of a width modulated micro-cavity filter with 20+20 designed half-holes a) Logarithmic scale, b) linear scale. The hole diameter was $180 \mathrm{~nm}$ and the cavity length was $1_{\mathrm{c}}=600 \mathrm{~nm}$.

By changing the cavity length $1_{c}$, in combination with the right choice of the lattice constant a, the peak wavelength of the filter can be tuned. For $1 \mathrm{D}$ photonic crystal micro-cavities ${ }^{9}$, the ratio between single order cavity length $\mathrm{l}_{\mathrm{c}}$ and lattice constant a should be around $l_{\mathrm{c}} / \mathrm{a}=1.5$. In accordance with this relation, the cavity length of the width modulated microcavities is designed around $585 \mathrm{~nm}$ for the chosen lattice constant of $390 \mathrm{~nm}$. Figure 5 and 6 show that the transmission peaks with these parameters are located in the center of the low transmission region of the filters. This proves that the rule for 1D photonic crystal micro-cavities is also applicable for the width modulated micro-cavities.

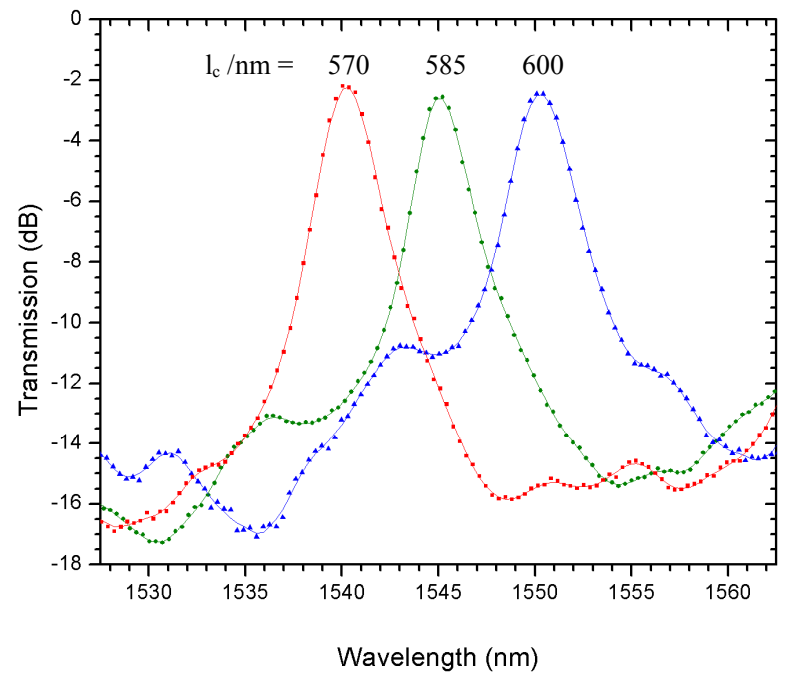

Figure 7. Transmission spectra of width modulated filters with three different cavity lengths $1_{\mathrm{c}}=570 \mathrm{~nm}$, $585 \mathrm{~nm}$, and $600 \mathrm{~nm}$. The designed half-hole diameters of the $20+20$ micro-cavities were $180 \mathrm{~nm}$.

Figure 7 shows transmission spectra of filters with different cavity lengths $1_{c}$. It is evident that an increasing cavity length will shift the filter peak to longer wavelengths. This behavior is shown for three cavity length $l_{\mathrm{c}}=570 \mathrm{~nm}, 585 \mathrm{~nm}$, and 
$600 \mathrm{~nm}$. The obtained wavelength shift per cavity length yields nearly constant $\Delta \lambda / \Delta \mathrm{l}_{\mathrm{c}}=0.33 \pm 0.05$ in the considered limited range of cavity lengths.

Furthermore, the center-wavelength of the transmission peak of the filters shifts if the designed half-hole diameter $d_{h h}$ and therefore the modulation depth change. The wavelength shift was determined to be $\Delta \lambda / \Delta d_{h h}=-0.98 \pm 0.01$.

\section{SUMMARY}

Design, fabrication, and optical characterization of 1-D micro-cavities in SOI waveguides fabricated in a CMOS environment using a $248 \mathrm{~nm}$ DUV lithography have been presented. The Bragg-mirror regions of the micro-cavities are formed by a modulation of the waveguide width. It was shown that the waveguide between designed adjacent half-holes is rounded by the fabrication process leading to a sinusoidal modulation of the waveguide width. Also the sub-micron spacer in the center of the images is rounded.

The transmission peak of a width modulated micro-cavity with 20 modulations in each of the two mirror regions $(20+20)$ and $180 \mathrm{~nm}$ designed half-hole diameter has a $-3 \mathrm{~dB}$ bandwidth of $1.8 \mathrm{~nm}$, which corresponds to a quality factor of $\mathrm{Q}=855$. The filter contrast was $13 \mathrm{~dB}$ for wavelengths $6 \mathrm{~nm}$ off the filter peak. The determined insertion loss of the filter was only $1.9 \mathrm{~dB}$.

\section{ACKNOWLEDGEMENT}

This work is supported by BMBF/VDI in the frame of project 13 N9732.

We thank Christian Meuer from Institut für Festkörperphysik of TU Berlin for his support.

\section{REFERENCES}

[1] Izhaky, N., Morse, M. T., Koehl, S., Cohen, O., Rubin, D., Barkai, A., Sarid, G., Cohen, R., Paniccia, M. J., "Development of CMOS-Compatible integrated silicon photonics devices", IEEE Journal of selected optics in quantum electronics 12(6), 1688 (2006).

[2] Reed, G., [Silicon Photonics: the state of the art], John Wiley \& Sons Ltd, England (2008).

[3] Noda, S., Chutinan, A., Imada, M., "Trapping and emission of photons by a single defect in a photonic bandgap structure", Nature 407, 608 (2000).

[4] Scheuera, J., Green, W. M. J., DeRose, G. A., Yariv, A., "Lasing from a circular Bragg nanocavity with an ultrasmall modal volume", Appl. Phys. Lett. 86, 251101 (2005).

[5] Dumon, P., Bogaerts, W., Wiaux, V., Wouters, J., Beckx, S., Van Campenhout, J., Taillaert, D., Luyssaert, B., Bienstman, P., Van Thourhout, D., Baets, R., "Low-loss SOI photonic wires and ring resonators fabricated with deep UV lithography", IEE photon. Technol. Lett. 16, 1328-1330 (2004).

[6] Foresi, J. S., Villeneuve, P. R., Ferrera, J.,Thoen, E. R., Steinmeyer, G. , Fan, S. , Joannopoulos, J. D. Kimerling, L. C., Smith, H. I., Ippen, E. P., "Photonic-bandgap micro-cavities in optical waveguides", Nature 390,143-135 (1997).

[7] Meister, S., Franke, B. A., Mahdi, S., Al-Saadi, A., Kuhlow, B., Voigt, K., Tillack, B., Richter, H. H., Zimmermann, L., Schrader, S., Ksianzou, V., Eichler, H. J., "Photonic crystal microcavities in SOI waveguides produced in a CMOS environment", Proc. SPIE 7606, 7606-41 (2010).

[8] Lefevre, H. C., "Single-mode fiber fractional wave devices and polarization controllers", Electronic Lett. 16, 778-780, (1980).

[9] Kuhlow, B., Przyrembel, G., Schlüter, S., Fürst, W., Steingrüber, R., Weimann, Ch., "Photonic Crystal Microcavities in SOI Photonic Wires for WDM Filter Applications", Journal of Lightwave Technology 25, 421431 (2007). 